\title{
Prognostic analysis of pancreatic carcinoma with portal system invasion following curative resection
}

\author{
Jing Wang", Shao-Cheng Lyu", Lin Zhou, Han Wang, Fei Pan, Tao Jiang, Ren Lang, Qiang He \\ Department of Hepatobiliary and Pancreatic Splenic Surgery, Beijing Chaoyang Hospital, Capital Medical University, Beijing, China \\ Contributions: (I) Conception and design: J Wang, SC Lyu; (II) Administrative support: R Lang, Q He; (III) Provision of study materials or patients: \\ J Wang, R Lang; (IV) Collection and assembly of data: J Wang, L Zhou, H Wang, F Pan, T Jiang; (V) Data analysis and interpretation: J Wang, SC \\ Lyu; (VI) Manuscript writing: All authors; (VII) Final approval of manuscript: All authors. \\ \#These authors contributed equally to this work. \\ Correspondence to: Prof. Ren Lang, Prof. Qiang He. No. 8 Gongtinan Road, Chaoyang District, Beijing 100020, China. Email: dr_langren@126.com; \\ heqiang349@sina.com.
}

Background: To analyze the related factors affecting the prognosis of pancreatic carcinoma with portal system invasion.

Methods: We retrospectively analyzed the clinical data of 118 patients with portal venous system invasion in Beijing Chaoyang Hospital between January 2011 and December 2018. Only patients with borderline resectable pancreatic cancer were included in this study. Borderline pancreatic cancer was defined according to NCCN (The National Comprehensive Cancer Network) guidelines. All patients underwent surgical treatment combined with vascular resection and reconstruction. The prognosis was evaluated according to the follow-up results, and the related risk factors for prognosis were analyzed. The survival curve was drawn by Kaplan-Meier method, and the survival rate was compared by log-rank test. Multivariate Cox regression was used to analyze the prognostic factors.

Results: In our research, all of 126 patients were successfully completed the operations. Complications occurred in $29.7 \%$ of patients and perioperative death in $4.0 \%$. A total of 118 patients were followed up and the followed-up rate was $97.5 \%$ (118/121). The overall 1-year, 2-year and 3-year survival rates were $49.2 \%, 27.1 \%$ and $19.8 \%$, And the median survival time was 20 months. Multivariate analysis showed that preoperative CA19-9 (RR 1.449, 95\% CI: 1.053-1.994), N status (RR 2.533, 95\% CI: 1.337-4.798), degree of tumor differentiation (RR 1.592, 95\% CI: 1.064-2.381) and venous invasion depth (RR 2.03, 95\% CI: 1.504-2.758) were independent risk factors for the prognosis.

Conclusions: The long-term prognosis of pancreatic carcinoma patients with portal system invasion is poor. The venous invasion depth is an independent risk factor for the prognosis of pancreatic carcinoma with portal system invasion, the deeper of venous invasion, the worse the prognosis, and poorly differentiated tumors have the worst prognosis. Other independent risk factors included $\mathrm{N}$ status and the preoperative CA19-9. Those may help with patients' selection for different treatment protocols.

Keywords: Pancreatic carcinoma; surgical procedures; vascular invasion; prognosis; risk factors

Submitted May 13, 2020. Accepted for publication Sep 18, 2020.

doi: $10.21037 /$ gs-20-495

View this article at: http://dx.doi.org/10.21037/gs-20-495 


\section{Introduction}

Due to the high degree of malignancy, easy occurrence of local vascular invasion and distant metastasis and other reasons, the overall resection rate of pancreatic cancer is less than $20 \%$, and the 5 -year survival rate is less than $10 \%$ (1). According to the latest international statistics, the incidence of pancreatic cancer in the world is the 14th malignant tumor, however, the death rate is the 6th (2). At the same time, some studies asserted that $17-32 \%$ of patients with pancreatic cancer have already had portal system (portal vein, superior mesenteric vein and splenic vein) invasion when diagnosed (3). Superior mesenteric vein $(\mathrm{SMV})$ and portal vein $(\mathrm{PV})$ invasion is frequent because of the proximity of these vessels to the uncinate process and pancreatic head. Potentially curative surgery is possible in these patients combining pancreatic resection with en bloc resection of the PV-SMV venous axis (4). Single-center reports, systematic reviews, and meta-analyses have shown the feasibility and the advantages of this approach, which may provide survival results comparable to those obtained with standard pancreatectomy without venous resection (5-8). Vascular invasion is considered to be one of the important causes of poor long-term prognosis in patients with pancreatic cancer. Other studies have stressed the role of histological venous invasion as prognostic factor, reporting worst survival in patients with venous invasion confirmed by pathological examination $(9,10)$. But the effect of the specific form of vascular invasion (including the location, depth, circumference, and mode of vascular reconstruction) on the prognosis is not clear. This paper reviews the clinical data of 118 pancreatic cancer patients with portal vein invasion treated in our center, in order to explore the effect of portal vein invasion on the long-term prognosis of the patients.

We present the following article in accordance with the STROBE reporting checklist (available at http://dx.doi. org/10.21037/gs-20-495).

\section{Methods}

\section{Ethics approval and consent to participate}

The study was conducted in accordance with the Declaration of Helsinki (as revised in 2013). The study was approved by the Ethics Committee of Beijing Chaoyang Hospital (No. 2019-D.-309-2). The participants provided written informed consent to participate in this study

\section{General clinical data}

The data of patients with pancreatic cancer treated in the Department of Hepatobiliary surgery of our hospital from January 2011 to December 2018 were analyzed retrospectively. according to the relevant inclusion criteria, a total of 118 patients with pancreatic cancer with portal vein invasion were selected.

Inclusion criteria: (I) patients with pancreatic cancer admitted to our hospital from January 2011 to December 2018; (II) preoperative imaging examination showed that there was or no invasion of portal vein system (portal vein, superior mesenteric vein and splenic vein); (III) complete resection of tumor and invaded blood vessels during operation; (IV) postoperative pathology confirmed pancreatic ductal adenocarcinoma (except pancreatic cystic tumor canceration, pancreatic acinar cell carcinoma, etc.); (V) the mode of operation and treatment strategy obtained the informed consent of patients and their families; (VI) the vascular replacement technique involved in the operation has been approved by the New Technology and Ethics Committee of our hospital and conforms to the regulations of ethics.

Exclusion criteria: (I) preoperative imaging examination showed that there was distant metastasis; (II) preoperative imaging examination showed that there was invasion of important celiac arteries (celiac trunk, common hepatic artery, abdominal aorta); (III) perioperative death; (IV) postoperative follow-up data were incomplete or lost to follow-up (Figure 1).

\section{Perioperative management}

All patients underwent radical surgery for pancreatic cancer. The scope of surgical resection is determined according to the location of the tumor (pancreaticoduodenectomy for pancreatic head and uncinate process tumors, pancreaticotail combined splenectomy for pancreatic body and tail tumors, and total pancreaticoduodenal combined splenectomy for tumors in the neck or wide range of pancreas). At present, there is no uniform clinical standard for the classification of vascular invasion in pancreatic cancer. The most commonly used clinical standard for vascular invasion is the Loyer classification and Shibata typing $(11,12)$. However, all of the above classifications have certain limitations. On the one hand, it is impossible to assess the site and scope of tumor invasion to portal vein system, on the other hand, it has 


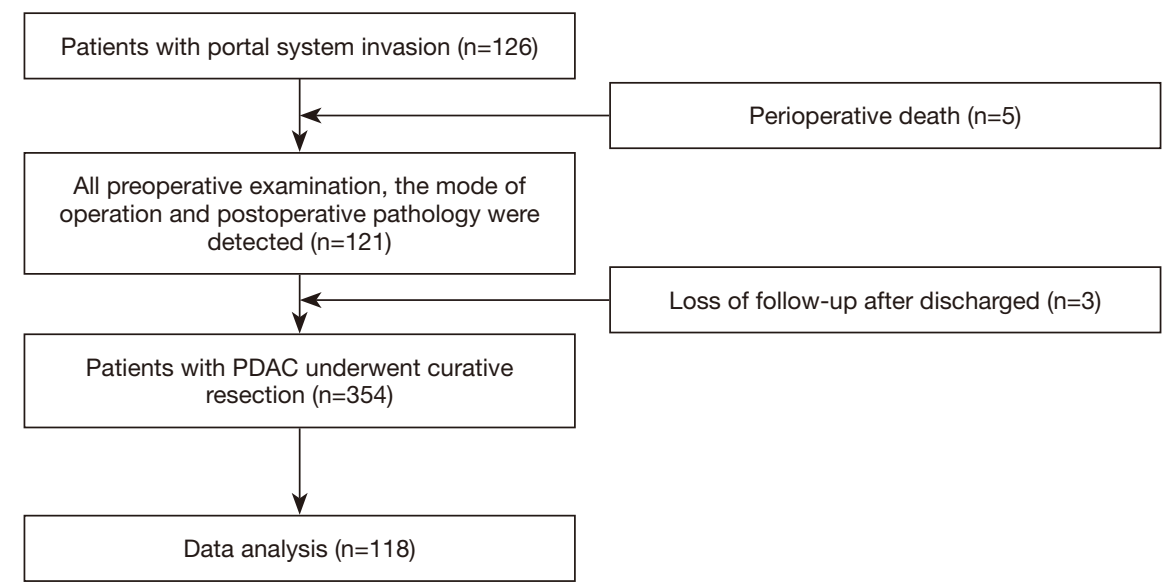

Figure 1 Screening flow chart.

no guiding value for the resection and reconstruction of the invaded portal vein system. In recent years, our center has carried out a beneficial attempt to optimize the above vascular invasion typing criteria in patients treated with radical surgery and proposed a new typing system (13). There are four types: (I) Portal and/or superior mesenteric vein invasions of less than 1/4-week diameter. In this type of patients, the lateral wall of the vein can be blocked without blocking the blood flow into the liver. The affected side wall can be partially excised and the vein can be sutured directly. After suturing, the vein can be guaranteed to have no obvious stenosis. (II) Portal vein and/or superior mesenteric vein were invaded to a range greater than $1 / 4$-week diameter, or the vein was clearly narrowed and occluded, without involving the splenic vein junction. In this type of patients, segmental resection of the involved vein is recommended, and end-to-end anastomosis or allograft or artificial vascular reconstruction is selected according to the tension of the upper and lower edges. (III) The tumor invaded the confluence of portal vein, splenic vein, and superior mesenteric vein. In this type of patients, partial splenic vein resection can be performed in conjunction with the confluence part, and splenic vein reconstruction can be completed by using foreign blood vessels with branches. (IV) The tumor invaded a wide area, the portal vein, splenic vein, and superior mesenteric vein are involved in the upper part, and the branch of superior mesenteric vein in the lower part is involved. In this type of patients, arterial approach is recommended to complete tumor dissociation and then resection of invaded vessels, for reconstruction, it is recommended that the superior mesenteric vein branch be shaped into an opening first, and then Allogeneic blood vessels with branches or other substitutes should be used to complete the reconstruction. Different methods of vascular resection and reconstruction are adopted according to the specific form of venous invasion. The technique of vascular reconstruction and the type of pancreatic, biliary, and enteric anastomoses depended on operating surgeon's choice. Cefoperazone sodium/sulbactam sodium was routinely used to prevent infection after operation. After operation, the gastric tube was removed according to the recovery of gastrointestinal function and diet was gradually restored, and the drainage tube was removed gradually according to the characteristics of drainage fluid and laboratory examination. on the premise of excluding the risk of postoperative bleeding, anticoagulation with low molecular weight heparin was used to prevent venous thrombosis after reconstruction. It is suggested that the patients should carry out follow-up chemotherapy in the department of oncology after discharged.

\section{Follow-up strategy}

Postoperative follow-up was performed by the combination of outpatient reexamination and telephone follow-up. The frequency of reexamination was as follows: 1 and 3 months after operation, once every 3 months within 2 years, and once every half a year for more than 2 years. The followup items mainly include: blood tests (blood routine, blood biochemistry, tumor marker) and imaging examinations (abdominal enhanced CT, Pulmonary CT). The contents of telephone follow-up mainly include: reexamination results, follow-up treatment, tumor recurrence and patients survival condition. 
Table 1 Surgical procedures performed in 118 patients submitted to pancreatectomy with $\mathrm{PV}$ or SMV resection for pancreatic adenocarcinoma

\begin{tabular}{|c|c|}
\hline Variable & Percentage, \% \\
\hline \multicolumn{2}{|l|}{ Procedure } \\
\hline Pancreaticoduodenectomy & 75.4 \\
\hline Total pancreatectomy & 15.3 \\
\hline $\begin{array}{l}\text { Radical resection of carcinoma of pancreatic } \\
\text { body and tail }\end{array}$ & 9.3 \\
\hline \multicolumn{2}{|l|}{ Length of surgery (h) } \\
\hline$\leq 10$ & 55.1 \\
\hline$>10$ & 44.9 \\
\hline \multicolumn{2}{|l|}{ Blood transfusion } \\
\hline Yes & 56.8 \\
\hline No & 43.2 \\
\hline \multicolumn{2}{|l|}{ Resected vein } \\
\hline Portal vein & 29.7 \\
\hline Superior mesenteric vein & 34.7 \\
\hline PV-SMV confluence & 35.6 \\
\hline \multicolumn{2}{|l|}{ Vein reconstruction mode } \\
\hline Direct suture & 9.3 \\
\hline End-to-end anastomosis & 22.9 \\
\hline Vascular replacement & 67.8 \\
\hline
\end{tabular}

$\mathrm{PV}$, portal vein, SMV, superior mesenteric vein.

\section{Extraction and analysis of related indicators}

The preoperative data of patients with venous invasion (sex, age, history of diabetes, treatment of reducing jaundice, tumor marker) were extracted from hospitalization and follow-up data. Intraoperative data (operation method, operation time, intraoperative blood loss, blood transfusion). Pathological data (tumor location, maximum tumor diameter, degree of differentiation, lymph node metastasis, cutting edge). Vascular invasion data (location, depth, circumference, and mode of vascular reconstruction) and follow-up data (postoperative chemotherapy, prognosis). According to the prognosis, the risk factors affecting the prognosis of patients with venous invasion were analyzed and the survival curve was drawn. Postoperative complications were defined according to the ISGPS. Postoperative mortality was defined as death occurring during the first 30 days after surgery or during hospitalization. Overall survival was calculated from the date of surgery to the date of death.

\section{Statistical analysis}

The measurement data are expressed by mean \pm standard deviation in accordance with normal distribution and by median (quartile spacing) in non-normal distribution. The survival curve was calculated and drawn by KaplanMeier method, and the survival rate was compared by Log-rank test. Cox proportional hazard model was used in multivariate analysis. The difference was statistically significant when $\mathrm{P}<0.05$, and all the data were analyzed by SPSS 22.0 software.

\section{Results}

\section{Patients' characteristics}

In the venous invasion group, there were 44 females and 74 males, female:male $=1: 1.7$. The age was 35 to $81(61.5 \pm 10.1)$ years old. 47 patients had a history of smoking. The main clinical manifestations were jaundice $(\mathrm{n}=60)$, abdominal pain $(n=44)$, gastrointestinal discomfort $(n=9)$ and physical examination $(\mathrm{n}=5)$. Among them, 17 patients underwent percutaneous transhepatic bile duct drainage because of jaundice before operation.

\section{Perioperative condition of patients with venous invasion}

All the 118 patients completed the operation successfully. Blood transfusion was used in 67 cases with intraoperative blood loss of $600 \mathrm{~mL}(400-1,000 \mathrm{~mL})$, and the operation time was 6-17 (11.7 \pm 2.3$)$ hours. According to the location of the tumor, radical pancreaticoduodenectomy was performed in 89 cases, total pancreatectomy in 18 cases, and radical resection of carcinoma of pancreatic body and tail in 11 cases. According to the specific forms of venous invasion, vascular replacement was performed in 80 cases after segmental resection, end-to-end anastomosis after segmental resection in 27 cases, and direct suture after wedge resection in 11 cases (Table 1).

\section{Postoperative outcomes}

All patients were discharged smoothly. The postoperative hospital stay was $6-58(20.8 \pm 9.7)$ days. Postoperative complications occurred in 35 cases $(29.7 \%)$, including 
Table 2 Postoperative morbidity of 118 patients submitted to pancreatectomy with $\mathrm{PV}$ or SMV resection for pancreatic adenocarcinoma

\begin{tabular}{|c|c|c|}
\hline Variable & Number & Percentage, \% \\
\hline Overall complications & 35 & 29.7 \\
\hline Pancreatic fistula & 9 & 7.62 \\
\hline Grade A & 4 & 3.39 \\
\hline Grade B & 4 & 3.39 \\
\hline Grade C & 1 & 0.84 \\
\hline DGE & 4 & 3.39 \\
\hline Biliary anastomotic leak & 5 & 4.24 \\
\hline Intestinal anastomotic leak & 4 & 3.39 \\
\hline Biochemical fistula & 10 & 8.47 \\
\hline Postoperative bleeding & 5 & 4.24 \\
\hline PV-SMV thrombosis & 2 & 1.69 \\
\hline Abdominal abscess & 9 & 7.63 \\
\hline Wound infection & 6 & 5.08 \\
\hline Urinary tract infection & 1 & 0.84 \\
\hline Cardiovascular complications & 1 & 0.84 \\
\hline DVT/PE & 2 & 1.69 \\
\hline Pancreatitis & 1 & 0.84 \\
\hline Pneumonia & 1 & 0.84 \\
\hline
\end{tabular}

DGE, delayed gastric emptying; DVT/PE, deep venous thrombosis/pulmonary embolism; PV, portal vein; SMV, superior mesenteric vein.

biochemical fistula $(\mathrm{n}=10)$, pancreatic fistula $(\mathrm{n}=5)$, biliary anastomotic leak $(n=4)$, Intestinal anastomotic leak $(n=4)$, abdominal abscess $(n=9)$, wound infection $(n=4)$, delayed gastric emptying $(n=4)$, postoperative bleeding $(n=5)$ and PV-SMV thrombosis $(\mathrm{n}=2)$, etc. (Table 2).

\section{Pathological analysis}

All patients were confirmed as pancreatic malignant tumor by pathological examination, accompanied by venous wall invasion. Among them, 111 patients achieved R0 resection, and the rate of $\mathrm{R} 0$ resection was $94.1 \%$. All of the 7 patients resected by $\mathrm{R} 1$ resection were positive for pancreatic amputation. The size of the tumor was 1.5-6.0 (3.5 \pm 1.0$)$ $\mathrm{cm}$. There were 84 patients with positive lymph nodes, with a positive rate of $71.2 \%$.

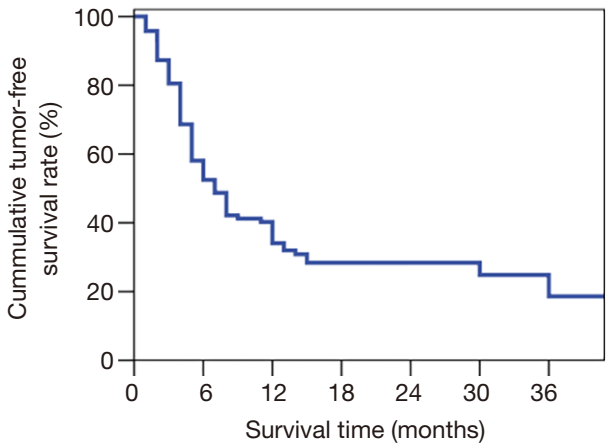

Figure 2 Disease-free survival curve of patients with venous invasion of pancreatic cancer.

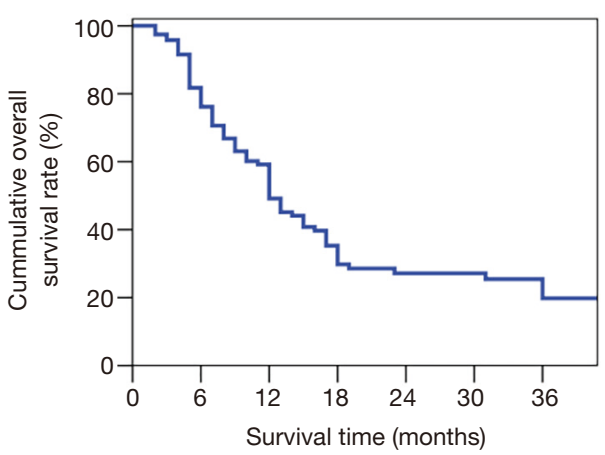

Figure 3 Overall survival curve of patients with venous invasion of pancreatic cancer.

\section{Prognosis of patients with venous invasion}

Up to April 2019, 118 patients were followed up, with a follow-up rate of $97.5 \%$. During the follow-up period, 47 patients received adjuvant chemotherapy with a chemotherapy cycle of 1 to 4 cycles, and at follow-up, 78 patients died of tumor recurrence, including 63 cases of liver, 12 cases of celiac lymph nodes and 3 cases of lung. The median disease-free survival time of patients with venous invasion was 17 months, and the disease-free survival rates of 1 year, 2 years and 3 years after operation were 34\%, 28.4\% and 18.6\%, respectively, as shown in Figure 2. And the median overall survival time was 20 months, and the overall survival rates of 1 year, 2 years and 3 years after operation were $49.2 \%, 27.1 \%$ and $19.8 \%$, respectively, as shown in Figure 3. 
Analysis of factors affecting the prognosis of patients with venous invasion

The results of Log-rank test showed that preoperative CA19-9 level, age, $\mathrm{N}$ status, intraoperative blood transfusion, depth of venous invasion, length of surgery and the degree of differentiation may be related to the prognosis of patients with venous invasion (Table 3). The above factors were included in the Cox proportional hazard model for multivariate analysis. Preoperative CA19-9 (RR 1.449, 95\% CI: $1.053-1.994), \mathrm{N}$ status (RR 2.533, 95\% CI: $1.337-4.798$ ), degree of tumor differentiation (RR 1.592, 95\% CI: 1.064-2.381) and venous invasion depth (RR 2.03, 95\% CI: 1.504-2.758) were independent risk factors for the prognosis of patients with venous invasion of pancreatic cancer (Table 4).

Table $3 \mathrm{Log}$-rank test analysis of prognosis in patients with venous invasion of pancreatic carcinoma

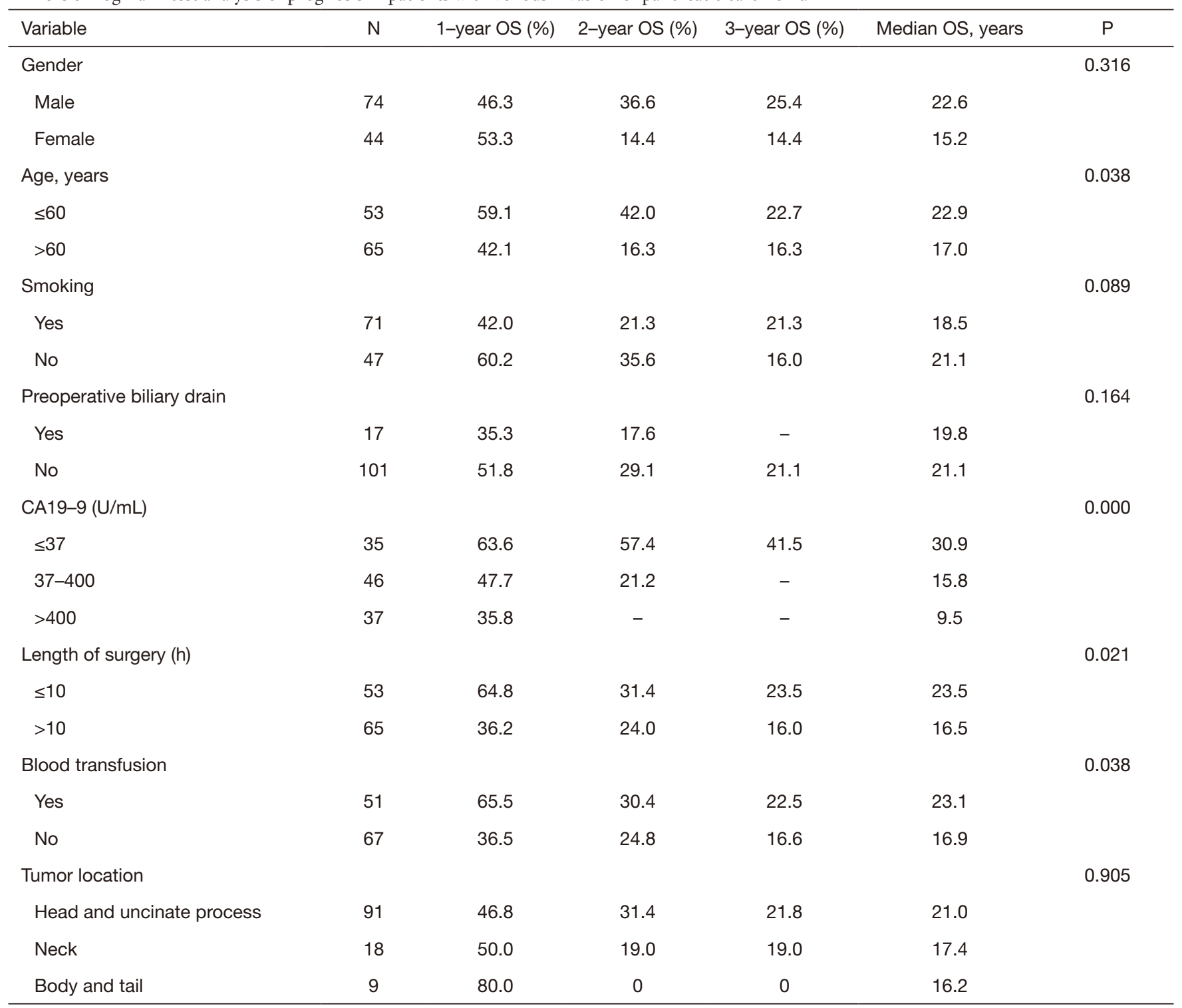

Table 3 (continued) 
Table 3 (continued)

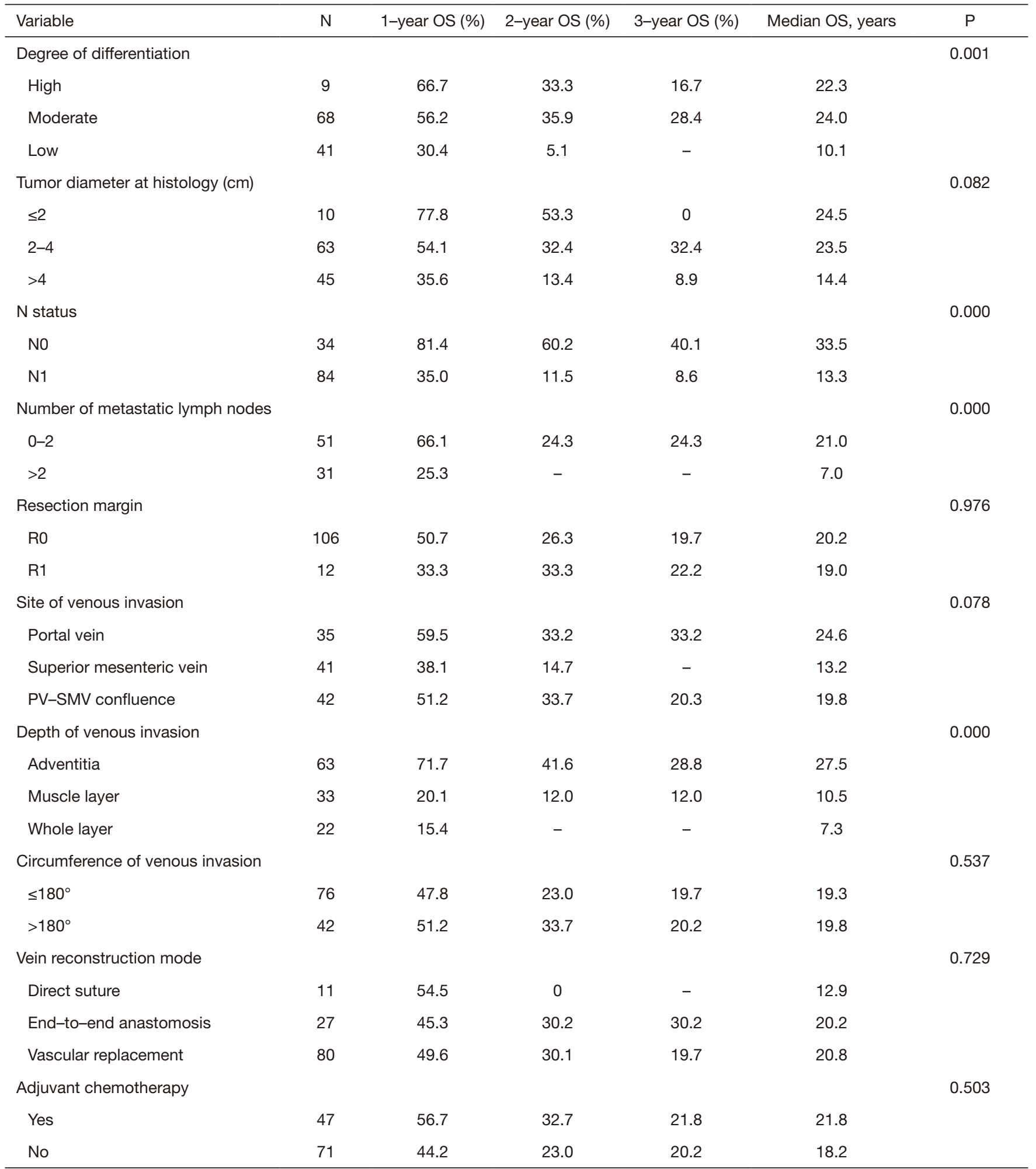

$\mathrm{N}$ nodal status according to the American Joint Committee on Cancer (AJCC) TNM staging of Pancreatic Cancer 2010. CA19-9, carbohydrate antigen 19-9; R, resection margin; PV, portal vein; SMV, superior mesenteric vein. 
Table 4 Multivariate proportional hazard regression (Cox model) analysis of prognosis in patients with venous invasion of pancreatic carcinoma

\begin{tabular}{lccc}
\hline Variable & $\mathrm{RR}$ & $95 \% \mathrm{Cl}$ & $\mathrm{P}$ \\
\hline CA19-9 & 1.449 & $1.053-1.994$ & 0.023 \\
Age & 1.008 & $0.611-1.663$ & 0.976 \\
Blood transfusion & 1.343 & $0.176-10.269$ & 0.776 \\
Depth of venous invasion & 2.037 & $1.504-2.758$ & 0.000 \\
$\mathrm{~N}$ status & 2.533 & $1.337-4.798$ & 0.004 \\
Degree of differentiation & 1.592 & $1.064-2.381$ & 0.024 \\
Length of surgery & 1.514 & $0.202-11.366$ & 0.687 \\
\hline
\end{tabular}

$\mathrm{RR}$, relative risk; $\mathrm{Cl}$, confidence interval.
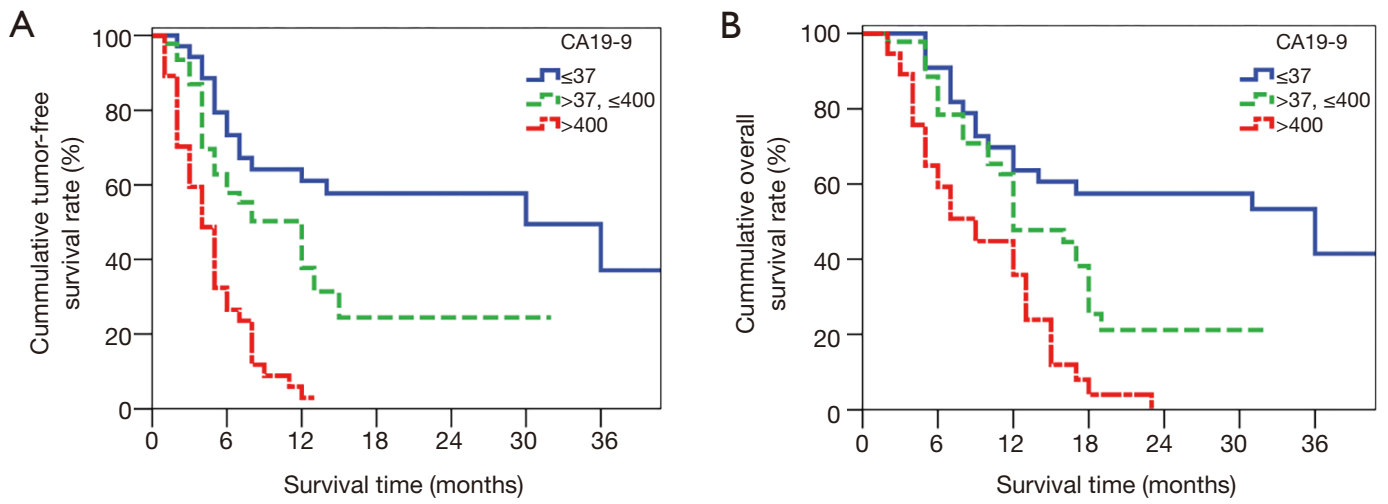

Figure 4 (A) DFS and (B) OS curves of patients with venous invasion at different preoperative levels of CA19-9.

\section{Stratified survival analysis of pancreatic cancer patients with venous invasion}

The first-, second- and third-year survival rates of patients with preoperative CA19-9 $\leq 37 \mathrm{U} / \mathrm{mL}$ were $63.6 \%, 57.4 \%$ and $41.5 \%$ respectively, those with $37 \mathrm{U} / \mathrm{mL}<$ preoperative CA19-9 $\leq 400 \mathrm{U} / \mathrm{mL}$ were $47.7 \%, 21.2 \%$ and 0 , respectively, and those of patients with preoperative CA19-9 $>400 \mathrm{U} / \mathrm{mL}$ were $35.8 \%, 0$ and 0 , respectively. The higher the level of CA19-9 before operation, the worse the prognosis of the patients. There was significant difference in the survival rate among the three groups $(\mathrm{P}=0.000$, Figure 4$)$. The first-, second- and third-year survival rates of patients without lymph node metastasis were $81.4 \%, 60.2 \%$ and $40.1 \%$, respectively, and those with lymphatic metastasis were $35 \%, 11.5 \%$, and $8.6 \%$, respectively, which means lymph node metastasis often predicts a poor prognosis. There was significant difference in the survival rate among the two groups ( $\mathrm{P}=0.001$, Figure 5$)$. As for the degree of differentiation, the first-, second- and third-year survival rates of patients with high degree were $66.7 \%, 33.3 \%$ and $16.7 \%$ respectively, those with moderate degree were $56.2 \%, 35.9 \%$ and $28.4 \%$, respectively, and those of patients with low degree were $30.4 \%, 5.1 \%$ and 0 , respectively. The lower the degree of differentiation, the worse the prognosis of the patients. There was significant difference in the survival rate among the three groups $(\mathrm{P}=0.001$, Figure 6). The first-, second- and third-year survival rates of patients with vein invasion to adventitia were $71.7 \%, 41.6 \%$ and $28.8 \%$, respectively, and those with vein invasion to muscle layer were $20.1 \%, 12.0 \%$ and $12.0 \%$, respectively, the patients with vein invasion to the whole layer were $15.4 \%$, 0 and 0 , respectively. The deeper the depth of venous invasion, the worse the prognosis of the patients, and the difference in survival rate among the three groups was statistically significant $(\mathrm{P}=0.000$, Figure 7$)$. The appearance of venous invasion at $40 \times, 100 \times$, and $200 \times$ microscopic at different layers are as follows (Figures 8,9,10). 

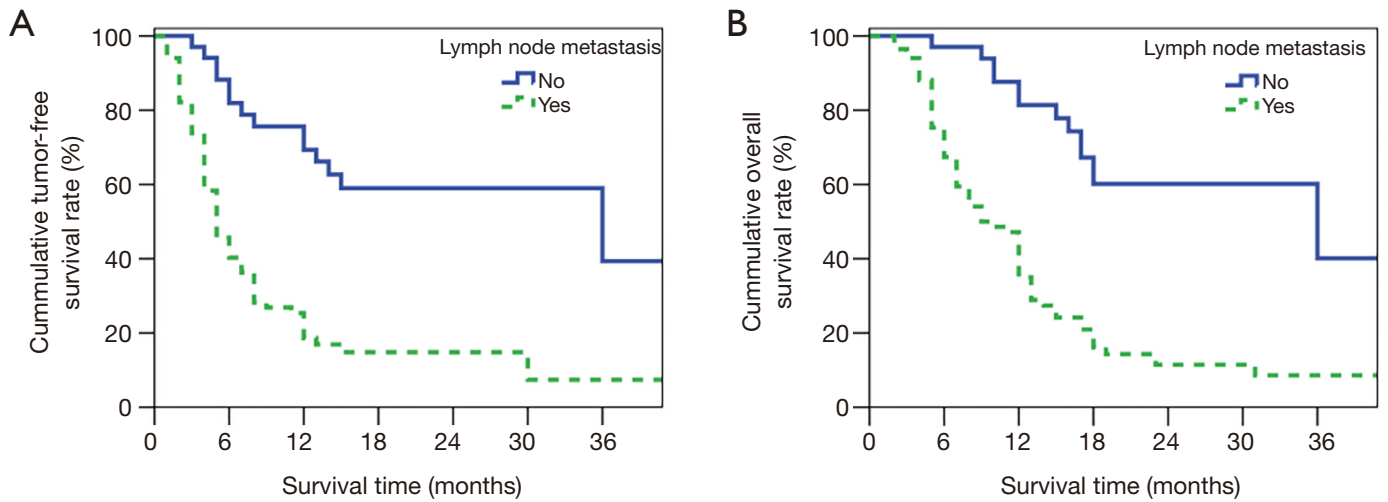

Figure 5 (A) DFS and (B) OS curves of patients with/without lymph node metastasis.
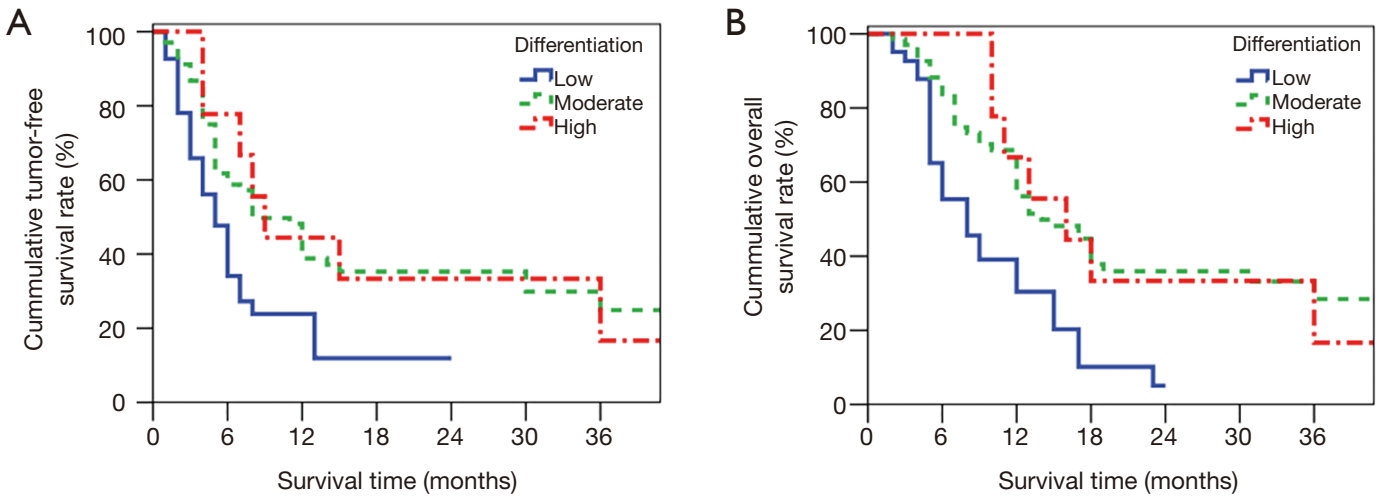

Figure 6 (A) DFS and (B) OS curves of patients with different degree of differentiation.
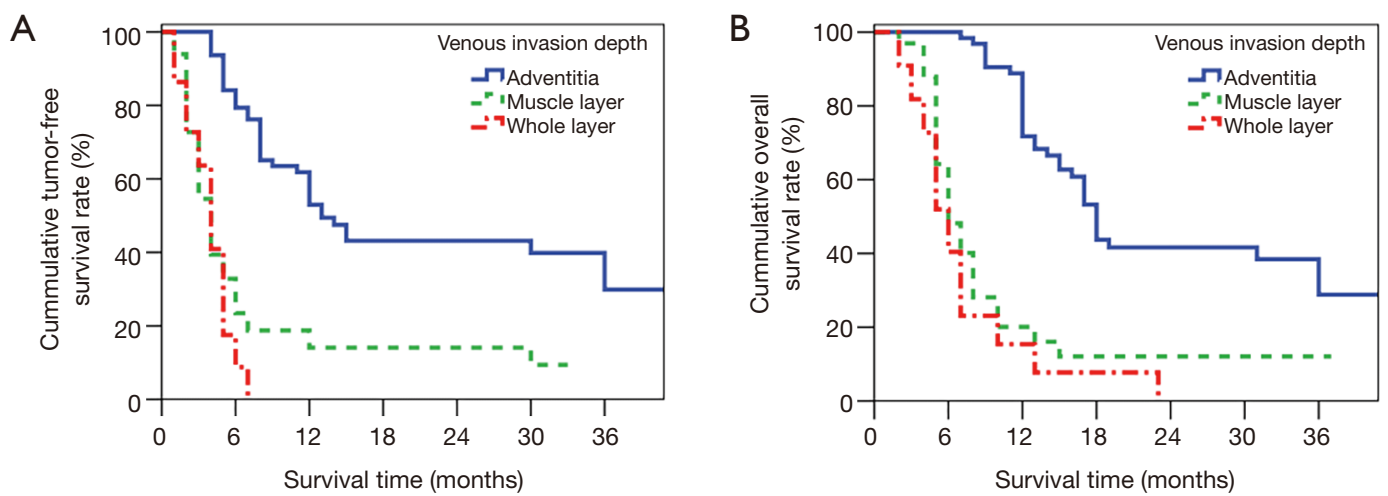

Figure 7 (A) DFS and (B) OS curves of patients with different vascular invasion depths. 

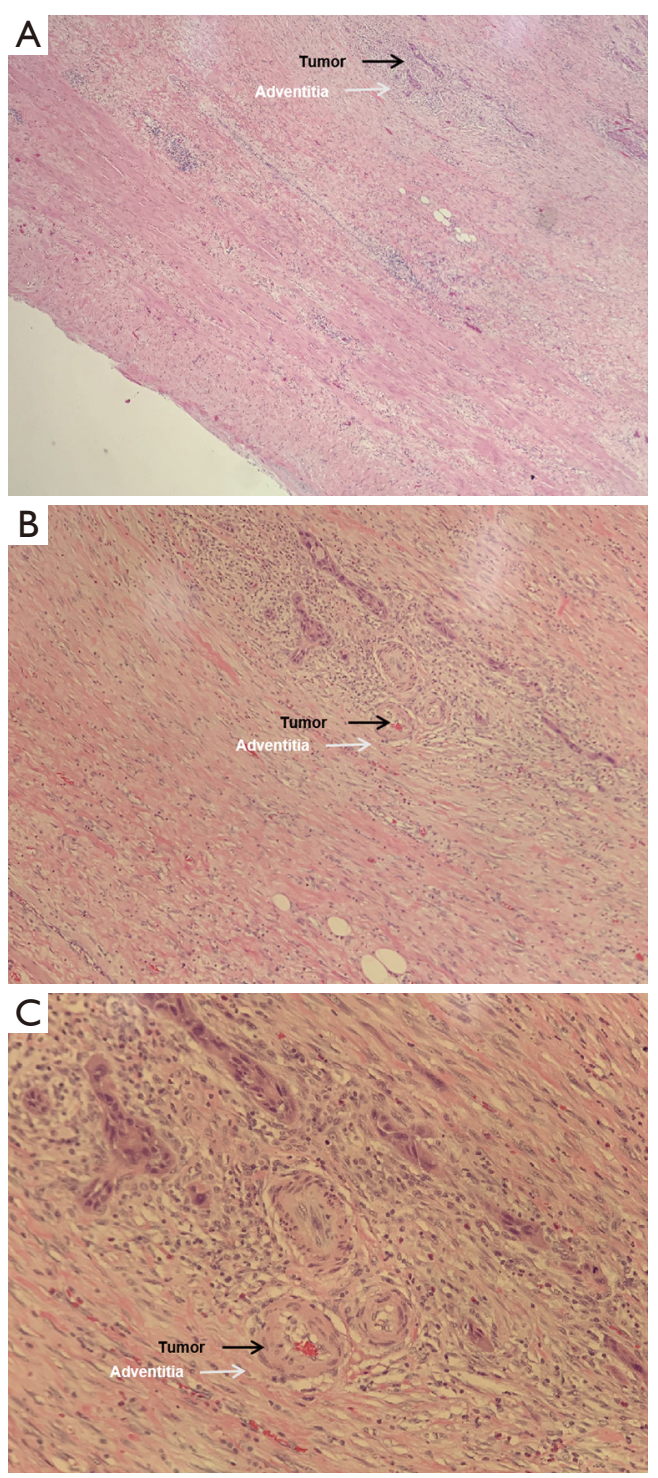

Figure 8 At (A) $40 \times$, (B) $100 \times$ and (C) $200 \times$ microscopic appearance of venous invasion into the adventitia (HE staining).

\section{Discussion}

Due to the adjacent structure of the surrounding tissue, pancreatic cancer may invade the portal vein system in the early stage of the tumor (14). Portal vein circulation is an important venous reflux system in human body. invasion of portal vein system will increase the difficulty of operation and the incidence of complications on the one hand, and promote tumor metastasis on the other hand. Kitagawa et al. (15) showed that the median survival time of pancreatic cancer patients with portal vein invasion was
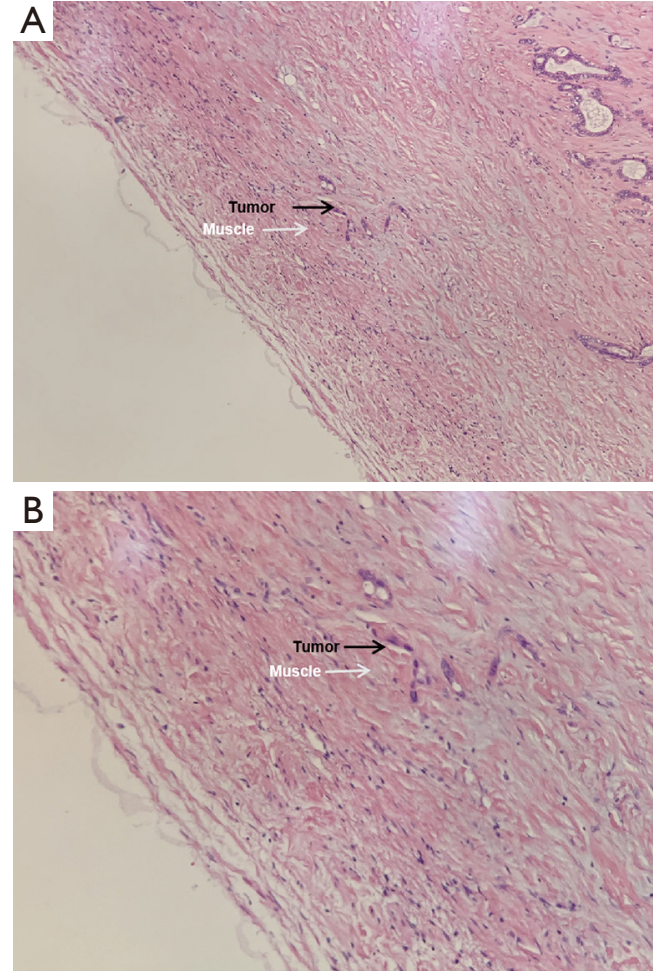

Figure 9 At (A) $100 \times$, (B) 200× microscopic appearance of venous invasion into the muscle layer (HE staining).

lower than that of patients without vascular invasion (22 vs. 31 months). Therefore, portal vein invasion is one of the important reasons for poor long-term prognosis of patients with pancreatic cancer.

As for the venous invasion of pancreatic cancer, at the end of the last century, due to the limitation of perioperative technology and adjuvant treatment, venous invasion has always been considered to be a taboo in the operation of pancreatic cancer. Since 2006, Prof. Varadhachary (16) first proposed the concept of borderline resectable pancreatic cancer, portal vein invasion is no longer a contraindication for pancreatic cancer surgery. Ravikumar et al. (17) retrospectively analyzed the data of patients from 9 pancreatic surgery centers in the UK, including 230 cases of combined vasectomy and 518 cases of palliative surgery. The median survival time of patients with combined vasectomy was 18.2 months, which was significantly better than that of palliative surgery ( 8 months). Therefore, surgery is of great significance in improving the prognosis of patients with pancreatic cancer invading the portal system. In terms of operation timing, in recent years, with the development 


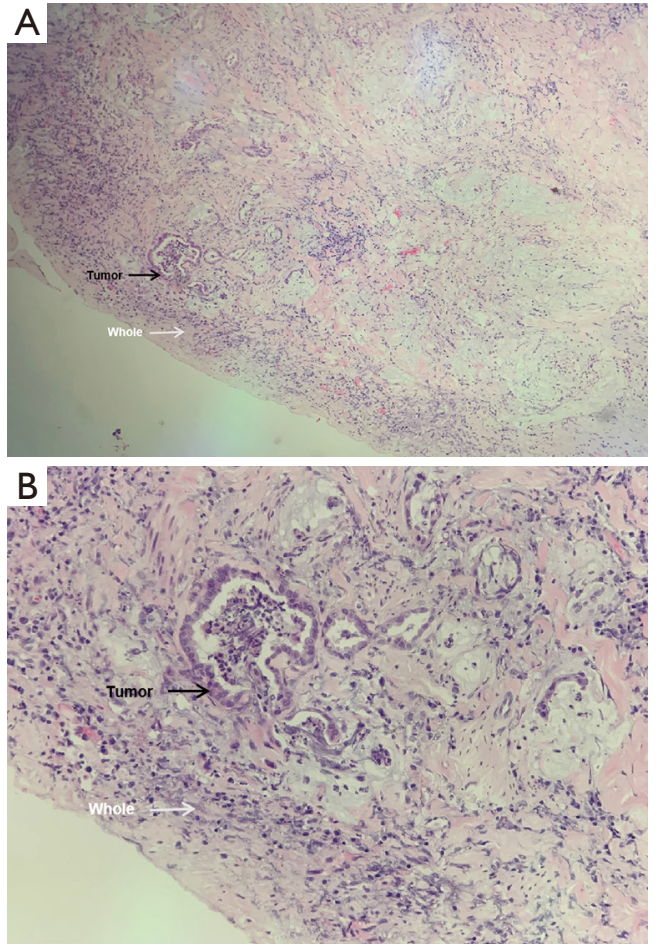

Figure 10 At (A) 100×, (B) 200× microscopic appearance of venous invasion into the whole layer (HE staining).

of preoperative neoadjuvant chemotherapy (18), the National Comprehensive Cancer Network guidelines of the United States have recommended that such patients should be treated with neoadjuvant therapy (19) before surgical resection since 2016. in order to improve the long-term prognosis of patients. However, some scholars still hold different views: the overall effective rate of chemotherapy for pancreatic cancer is less than $30 \%$, and even the highly toxic FOLFIRINOX regimen combined with four drugs is only about $50 \%$ (20), while the effect of this regimen is even worse for Chinese patients (21). If neoadjuvant chemotherapy is used first, it may also cause delays in the timing of surgery. Therefore, the study of the risk factors affecting the surgical prognosis of patients with pancreatic cancer invaded by the portal vein system has a certain guiding significance for clinical decision-making of the first choice of treatment.

For the risk factors of long-term prognosis of patients with pancreatic cancer with portal vein invasion, this study shows that preoperative CA19-9 level, N status, degree of differentiation and the depth of vein invasion are independent risk factors. In recent years, great progress has been made in molecular and serology of pancreatic cancer, but CA19-9 is still an important serological marker for clinical diagnosis and prognosis of pancreatic cancer (22). Sugiura et al. (23) by reviewing the data of 154 patients with pancreatic cancer, preoperative CA19-9 was an independent risk factor for long-term prognosis of patients with pancreatic cancer, and the median survival time of patients in the low level group was significantly better than that in the high level group (31 vs. 16 months). Boeck et al. (24) through a systematic review of the literature, it is concluded that CA19-9 is an important serological marker for judging the prognosis and monitoring of patients with pancreatic cancer, and its decreasing level can also reflect the effect of treatment to some extent.

However, there are relatively few studies on the effects of the specific forms of portal vein invasion (including the location, depth, circumference, and revascularization mode of vein invasion) on the long-term prognosis of pancreatic cancer. Ramacciato et al. (25) reviewed the data of 406 patients undergoing pancreatic cancer surgery in 8 pancreatic centers in Italy. Multivariate analysis confirmed that pancreatic cancer portal vein invasion was an independent risk factor for poor prognosis. The median survival time and 5 -year survival rate in the vascular invasion group were significantly worse than those in the non-vascular invasion group (20 vs. 33 months; $20 \%$ vs. $33.7 \%)$. These results confirm the important role of venous invasion as prognostic factors, as reported by others (26-28). However, other studies did not detect the prognostic role of venous invasion, but the small number of included patients limits them $(29,30)$. At the same time, it also showed that the specific location and circumference of vascular invasion had no significant correlation with the prognosis, which were the same as our research. The data of our study show that the depth of vascular invasion is an independent risk factor for prognosis. we think that when the tumor invades the whole layer of the blood vessel wall, the tumor cells are more likely to fall off into the blood, enter the portal vein system circulation, and occur blood metastasis. this kind of patients have early postoperative tumor recurrence and poor prognosis.

As for the $\mathrm{N}$ status, with univariate analysis, we found a significant correlation between the prognosis of patients with portal venous system invasion and lymph nodal diffusion and the number of metastatic nodes. Current studies have revealed that the abundant lymphatic circulatory system in the peripancreatic retroperitoneum is the most accessible organ (sentinel organ) of pancreatic 
metastatic cancer cells, and lymphatic metastasis may occur in the early stage of the formation of primary lesion formation. It has extensive communication with the accompanying blood circulation and neural network, and has the potential to incubate, culture, transmit and hide metastatic cancer cells. It is speculated that it plays an important role in the invasion and metastasis of pancreatic cancer, corroborating the hypothesis that venous invasion is not only a consequence of the intimate anatomic relationship between the pancreas and the PV/SMV axis, but also a sign of aggressiveness of the disease (31). In the light of these findings, further studies might add careful preoperative evaluation of radiological nodal status to tumor/vein interface analysis at CT in an effort to better predict the presence of pathological venous invasion. However, we did not include the number of lymph node metastases in the multivariate proportional hazard regression (Cox model) analysis of prognosis in patients with venous invasion of pancreatic carcinoma, this is because the total number of lymph nodes obtained during surgery is different depending on the location of tumor and the surgical method, the assessment of the number of metastatic nodes may bias the result.

The pathological features of the tumor include the degree of differentiation of the tumor, nerve invasion, vascular tumor thrombus and so on. Its effect on the prognosis of patients with pancreatic ductal adenocarcinoma has been mentioned in a large number of previous studies, but no consensus has been reached at present. At present, there is no unified standard for judging the differentiation degree of pancreatic ductal adenocarcinoma, and there are two widely used: The first is that WHO proposed a grading system similar to that of gastrointestinal adenocarcinoma. The cancer tissues with high differentiation, $\geq 90 \%$ are glandular duct structure; moderately differentiated, 50-90\% are glandular tube structure; poorly differentiated, cancer nest tissue or single cancer cell $>50 \%$ (32). The other is to show the heterogeneity of the tumor by the score of infiltration: well-differentiated glandular ducts, 1 point; poorly differentiated glandular ducts, 2 points; no glandular structure formation (including solid, cord-like, single cancer cells, etc.), 3 points. The main and secondary components are added together to get a total score (level $1<4$ points, level $2=4$ points, level $3>4$ points) (33). The first evaluation method is used in our research. The relationship between tumor differentiation and prognosis has been reported in many literatures. most of the literatures have concluded that pancreatic carcinoma with low differentiation is more likely to have recurrence and metastasis than well-differentiated pancreatic carcinoma, and has a shorter survival time $(34,35)$. In 2013, Matthew published an article that the degree of tumor differentiation has the same influence on the evaluation of prognosis as the status of lymph node metastasis, and proposed the TNMG staging system, which includes the degree of tumor differentiation into the staging system, and pointed out that this system can better stratify the prognosis of patients with pancreatic cancer $(36,37)$. In this study, with multivariate analysis, the degree of tumor differentiation was considered to be an independent prognostic risk factor for OS, which was consistent with the results reported in the previous literature. Therefore, for patients with portal vein system invasion, it is not enough to judge after prognosis by TNM staging alone. It is necessary to further judge the heterogeneity of different tumors on the basis of TNM staging, and take specific therapeutic effects for different degrees of differentiation of pancreatic ductal adenocarcinoma in order to achieve a better prognosis.

Our result shows that the depth of vascular invasion is an important independent prognostic factor for pancreatic cancer patients with portal vein invasion. In our perspective, the deeper the vascular invasion is, the more likely the tumor cells will break through the vascular wall. In this way, the tumor cells can easily enter the portal vein circulation and have early distant metastasis, which is also an important reason for the poor long-term prognosis of patients. Therefore, for patients considered to have portal vein invasion before surgery, the depth of invasion can be preliminarily determined through enhanced imaging. If the continuity of portal vein wall is interrupted or there is a filling defect in the lumen, the depth of vascular invasion should be fully evaluated at this time and the operation should be carefully considered. As for patients undergoing surgical resection, we also suggest that the depth of vascular wall invasion should be judged routinely. If the entire layer is invaded, regular chemotherapy should be implemented as soon as possible after the operation, and follow-up strategies should be strengthened. Similarly, our research also shows that the higher the value of CA19-9, the worse the tumor differentiation and lymph node metastasis are also independent risk factors affecting the prognosis of patients. Therefore, regular chemotherapy should be implemented for such patients as well.

In addition, this study showed that whether the postoperative incisal margin is positive or not had no significant correlation with the long-term prognosis of 
the patients. For the case of incisal margin, there may be a certain bias because only 12 patients in this study have positive incisal margin. At present, the prognostic role of resection margin in patients with borderline resectable tumors was demonstrated only by some studies, whereas in other studies it was not a significant prognostic factor for overall survival. The complex relationship between histologic venous invasion, venous resection, and negative resection margins should be further studied and explained.

\section{Conclusions}

To sum up, the long-term prognosis of pancreatic carcinoma patients with portal venous system invasion is poor. The venous invasion depth is an independent risk factor for the prognosis of pancreatic carcinoma with portal venous system invasion, the deeper of venous invasion, the worse the prognosis, and poorly differentiated tumors have the worst prognosis. Other independent risk factors included $\mathrm{N}$ status and the preoperative CA19-9. Those may help with patients' selection for different treatment protocols.

\section{Acknowledgments}

Funding: This study was supported by the Beijing Municipal Science \& Technology Commission, China (Z181100001718164).

\section{Footnote}

Reporting Checklist: The authors have completed the STROBE reporting checklist. Available at http://dx.doi. org/10.21037/gs-20-495

Data Sharing Statement: Available at http://dx.doi. org/10.21037/gs-20-495

Conflicts of Interest: All authors have completed the ICMJE uniform disclosure form (available at http://dx.doi. org/10.21037/gs-20-495). The authors have no conflicts of interest to declare.

Ethical Statement: The authors are accountable for all aspects of the work in ensuring that questions related to the accuracy or integrity of any part of the work are appropriately investigated and resolved. The study was conducted in accordance with the Declaration of Helsinki (as revised in 2013). The study was approved by the Ethics
Committee of Beijing Chaoyang Hospital (No. 2019-D.309-2). The participants provided written informed consent to participate in this study.

Open Access Statement: This is an Open Access article distributed in accordance with the Creative Commons Attribution-NonCommercial-NoDerivs 4.0 International License (CC BY-NC-ND 4.0), which permits the noncommercial replication and distribution of the article with the strict proviso that no changes or edits are made and the original work is properly cited (including links to both the formal publication through the relevant DOI and the license). See: https://creativecommons.org/licenses/by-nc-nd/4.0/.

\section{References}

1. Bray F, Ferlay J, Soerjomataram I, et al. Global cancer statistics 2018: GLOBOCAN estimates of incidence and mortality worldwide for 36 cancers in 185 countries. CA Cancer J Clin 2018;68:394-424.

2. Siegel RL, Miller KD, Jemal A. Cancer statistics, 2018. CA Cancer J Clin 2018;68:7-30.

3. Egawa S, Toma H, Ohigashi H, et al. Japan Pancreatic Cancer Registry;30th year anniversary: Japan Pancreas Society. Pancreas 2012;41:985-92.

4. Tseng JF, Tamm EP, Lee JE, et al. Venous resection in pancreatic cancer surgery. Best Pract Res Clin Gastroenterol 2006;20:349-64.

5. Harrison LE, Klimstra DS, Brennan MF. Isolated portal vein involvement in pancreatic adenocarcinoma. A contraindication for resection? Ann Surg 1996;224:342-7; discussion 347-9.

6. Fuhrman GM, Leach SD, Staley CA, et al. Rationale for en bloc vein resection in the treatment of pancreatic adenocarcinoma adherent to the superior mesentericportal vein confluence. Pancreatic Tumor Study Group. Ann Surg 1996;223:154-62.

7. Ramacciato G, Mercantini P, Petrucciani N, et al. Does portal-superior mesenteric vein invasion still indicate irresectability for pancreatic carcinoma? Ann Surg Oncol 2009;16:817-25.

8. Zhou Y, Zhang Z, Liu Y, et al. Pancreatectomy combined with superior mesenteric vein-portal vein resection for pancreatic cancer: a meta-analysis. World J Surg 2012;36:884-91.

9. Fukuda S, Oussoultzoglou E, Bachellier P, et al. Significance of the depth of portal vein wall invasion after curative resection for pancreatic adenocarcinoma. Arch 
Surg 2007;142:172-9; discussion 180.

10. Wang J, Estrella JS, Peng L, et al. Histologic tumor involvement of superior mesenteric vein/portal vein predicts poor prognosis in patients with stage II pancreatic adenocarcinoma treated with neoadjuvant chemoradiation. Cancer 2012;118:3801-11.

11. Kahl S, Glasbrenner B, Zimmermann S, et al. Endoscopic ultrasound in pancreatic diseases. Dig Dis 2002;20:120-6.

12. Illuminati G, Carboni F, Lorusso R, et al. Results of a pancreatectomy with a limited venous resection for pancreatic cancer. Surg Today 2008;38:517-23.

13. Zhu J, Li X, Kou J, et al. Proposed Chaoyang vascular classification for superior mesenteric-portal vein invasion, resection, and reconstruction in patients with pancreatic head cancer during pancreaticoduodenectomy - A retrospective cohort study. Int J Surg 2018;53:292-297.

14. Bockhorn M, Uzunoglu FG, Adham M, et al. Borderline resectable pancreatic cancer: a consensus statement by the International Study Group of Pancreatic Surgery (ISGPS). Surgery 2014;155:977-88.

15. Kitagawa H, Tajima H, Nakagawara H, et al. En bloc vascular resection for the treatment of borderline resectable pancreatic head carcinoma. Mol Clin Oncol 2014;2:369-74.

16. Varadhachary GR, Tamm EP, Abbruzzese JL, et al. Borderline resectable pancreatic cancer: definitions, management, and role of preoperative therapy. Ann Surg Oncol 2006;13:1035-46.

17. Ravikumar R, Sabin C, Abu HM, et al. Impact of portal vein infiltration and type of venous reconstruction in surgery for borderline resectable pancreatic cancer. Br J Surg 2017;104:1539-48.

18. Katz MH, Shi Q, Ahmad SA, et al. Preoperative Modified FOLFIRINOX Treatment Followed by CapecitabineBased Chemoradiation for Borderline Resectable Pancreatic Cancer: Alliance for Clinical Trials in Oncology Trial A021101. JAMA Surg 2016;151:e161137.

19. Dhir M, Malhotra GK, Sohal D, et al. Neoadjuvant treatment of pancreatic adenocarcinoma: a systematic review and meta-analysis of 5520 patients. World J Surg Oncol 2017;15:183.

20. Conroy T, Gavoille C, Samalin E, et al. The role of the FOLFIRINOX regimen for advanced pancreatic cancer. Curr Oncol Rep 2013;15:182-9.

21. Bai X, Su R, Ma T, et al. Modified FOLFIRINOX for advanced pancreatic cancer: a tertiary center experience from China. Zhonghua Wai Ke Za Zhi 2016;54:270-5.

22. Le N, Sund M, Vinci A. Prognostic and predictive markers in pancreatic adenocarcinoma. Dig Liver Dis 2016;48:223-30.

23. Sugiura T, Uesaka K, Kanemoto H, et al. Serum CA199 is a significant predictor among preoperative parameters for early recurrence after resection of pancreatic adenocarcinoma. J Gastrointest Surg 2012;16:977-85.

24. Boeck S, Stieber P, Holdenrieder S, et al. Prognostic and therapeutic significance of carbohydrate antigen 19-9 as tumor marker in patients with pancreatic cancer. Oncology 2006;70:255-64.

25. Ramacciato G, Nigri G, Petrucciani N, et al. Pancreatectomy with Mesenteric and Portal Vein Resection for Borderline Resectable Pancreatic Cancer: Multicenter Study of 406 Patients. Ann Surg Oncol 2016;23:2028-37.

26. Nakagohri T, Kinoshita T, Konishi M, et al. Survival benefits of portal vein resection for pancreatic cancer. Am J Surg 2003;186:149-53.

27. Kurihara C, Yoshimi F, Sasaki K, et al. Impact of portal vein invasion and resection length in pancreatoduodenectomy on the survival rate of pancreatic head cancer. Hepatogastroenterology 2013;60:1759-65.

28. Boggi U, Del CM, Croce C, et al. Prognostic implications of tumor invasion or adhesion to peripancreatic vessels in resected pancreatic cancer. Surgery 2009;146:869-81.

29. Jeong J, Choi DW, Choi SH, et al. Long-term outcome of portomesenteric vein invasion and prognostic factors in pancreas head adenocarcinoma. ANZ J Surg 2015;85:264-9.

30. Carrère N, Sauvanet $A$, Goere D, et al. Pancreaticoduodenectomy with mesentericoportal vein resection for adenocarcinoma of the pancreatic head. World J Surg 2006;30:1526-35.

31. Rehders A, Stoecklein NH, Guray A, et al. Vascular invasion in pancreatic cancer: tumor biology or tumor topography? Surgery 2012;152:S143-51.

32. Lüttges J, Schemm S, Vogel I, et al. The grade of pancreatic ductal carcinoma is an independent prognostic factor and is superior to the immunohistochemical assessment of proliferation. J Pathol 2000;191:154-61.

33. Adsay NV, Basturk O, Bonnett M, et al. A proposal for a new and more practical grading scheme for pancreatic ductal adenocarcinoma. Am J Surg Pathol 2005;29:724-33.

34. Yan L, Siddiqui AA, Laique SN, et al. A large multicenter study of recurrence after surgical resection of branch-duct intraductal papillary mucinous neoplasm of the pancreas. Minerva Gastroenterol Dietol 2017;63:50-54.

35. Lim JE, Chien MW, Earle CC. Prognostic 
factors following curative resection for pancreatic adenocarcinoma: a population-based, linked database analysis of 396 patients. Ann Surg 2003;237:74-85.

36. Wasif N, Ko CY, Farrell J, et al. Impact of tumor grade on prognosis in pancreatic cancer: should we include grade in

Cite this article as: Wang J, Lyu SC, Zhou L, Wang H, Pan F, Jiang T, Lang R, He Q. Prognostic analysis of pancreatic carcinoma with portal system invasion following curative resection. Gland Surg 2021;10(1):35-49. doi: 10.21037/gs-20495
AJCC staging? Ann Surg Oncol 2010;17:2312-20.

37. Rochefort MM, Ankeny JS, Kadera BE, et al. Impact of tumor grade on pancreatic cancer prognosis: validation of a novel TNMG staging system. Ann Surg Oncol 2013;20:4322-9. 